\title{
Singularities in large deviations of work in quantum quenches
}

\author{
P. Rotondo, ${ }^{1,2}$ J. Mináŕ, ${ }^{1,2,3}$ J. P. Garrahan, ${ }^{1,2}$ I. Lesanovsky, ${ }^{1,2}$ and M. Marcuzzi ${ }^{1,2}$ \\ ${ }^{1}$ School of Physics and Astronomy, University of Nottingham, Nottingham, NG7 2RD, UK \\ ${ }^{2}$ Centre for the Mathematics and Theoretical Physics of Quantum Non-equilibrium Systems, \\ University of Nottingham, Nottingham NG7 2RD, UK \\ ${ }^{3}$ Department of Physics, Lancaster University, Lancaster, LA1 4YB, UK
}

\begin{abstract}
We investigate large deviations of the work performed in a quantum quench across two different phases separated by a quantum critical point, using as an example the Dicke model quenched from its superradiant to its normal phase. We extract the distribution of the work from the Loschmidt amplitude and compute for both the corresponding large-deviation forms. Comparing these findings with the predictions of the classification scheme put forward in [Phys. Rev. Lett. 109, 250602 (2012)], we are able to identify a regime which is in fact distinct to the ones identified so far: here the rate function exhibits a non-analytical point which is a strong indication of the existence of an out-of-equilibrium phase transition in the rare fluctuations of the work.
\end{abstract}

\section{INTRODUCTION}

Understanding out-of-equilibrium phenomena in classical and quantum many-body systems is one of the modern challenges in condensed matter and statistical physics. The breakdown of equilibrium conditions, associated to the absence of detailed balance in the microscopic processes governing the dynamics, results in asymptotic states which do not take the equilibrium Boltzmann-Gibbs form. Some concepts and techniques from thermodynamics and statistical mechanics can be however transferred to an out-of-equilibrium regime, leading for instance to fluctuation-dissipation relations (which connect the response of a system under a weak external perturbation to the correlation between equilibrium thermal fluctuations, see, e.g., ${ }^{1 / 2}$ ) and fluctuation ${ }^{3}$ relations.

In closed quantum systems, the simplest conceptual protocol to obtain an out-of-equilibrium evolution is a quantum quench. Physically, this can be thought of as an abrupt change $\Omega_{0} \rightarrow \Omega$ of one of the external fields appearing in the Hamiltonian $H$, fast enough for the state of the system not to appreciably change across its variation. Typically, one starts from the ground state $\left|\mathrm{GS}\left(\Omega_{0}\right)\right\rangle$ of $H\left(\Omega_{0}\right)$ before the quench (time $t \rightarrow 0^{-}$) and subsequently evolves it for $t>0$ with $H(\Omega)$. Such quantum quenches have been extensively studied to understand relaxation and thermalization in closed quantum systems 455 and their relation to integrability, both theoretically ${ }^{6 / 7}$ and experimentally $\sqrt{8[10}$.

Interestingly, the notion of work can be generalized to the quantum regime and fluctuation relations have been found to hold much like they do in a classical context $t^{11 / 12}$. Furthermore, it has been established that the Loschmidt amplitude $L(t)$ for a quenched system satisfies, in the thermodynamic limit $N \rightarrow \infty$, a large deviation principle $L(t) \sim e^{N l(t)}$. The analytical continuation of $l(t)$ to imaginary time $t \rightarrow-i s$ is related (via a Legendre transform) to the statistics of the work done on the system by the quench 13 . The function $l(-i s)$ is typically referred to as scaled cumulant generating function (SCGF for short).
Gambassi and Silva ${ }^{14}$ provided a first classification of the possible forms of these large deviation functions, identifying two distinct kinds of qualitative behaviors: for systems in class A (spectrum bounded from above), the SCGF is defined for all values of $s \in \mathbb{R}$, whereas for systems in class B (spectrum unbounded from above) the SCGF is defined only for values of $s$ larger than a certain threshold value $s>s^{*}$.

In this work we shed light on the behavior of large fluctuations in the work performed during a quench across a quantum critical point. We show that the statistics of the work may exhibit a non-analytical point, corresponding to a non-equilibrium phase transition, a situation encountered in the studies of the rare events of out-of-equilibrium classical stochastic systems $\frac{15 / 16}{}$. Importantly, this constitutes a novel feature of the statistics of the work fluctuations not included in the classification scheme put forward in Ref ${ }^{[14}$. For the sake of concreteness we illustrate our ideas using the Dicke mode ${ }^{17}$, a paradigmatic Hamiltonian of light-matter interaction. In the past decade, extensive investigations addressed its implementation $\frac{18}{18}$ and connection to the lowenergy physics of Bose-Einstein condensates in optical cavities $^{19}$, its hallmark superradiant phase transition 20121 (experimentally probed in 22 24), the associated critical phenomena ${ }^{25 \mid 27}$ and non-equilibrium properties 28129 , its connection to the physics of spin glasses $30 \mid 32$ and neural networks $s^{33 \mid 34}$ and its application in the context of the self-organization of the atomic motion 35 .

Exploiting the inherent integrability of this model in the thermodynamic limit 20212 to construct an explicit, though approximate, representation for the distribution of the work, we highlight a parameter regime going beyond the classification proposed in ${ }^{14}$ and the corresponding structure of the rate function. Conceptually, we proceed by: (i) establishing a convenient formalism to describe the quench protocol we address; (ii) extracting the large-deviation form of the Loschmidt amplitude in the thermodynamic limit; (iii) highlighting the emergence of a point of non-analyticity in the corresponding rate function describing the fluctuations of the work. 


\section{THE MODEL}

We start by setting the notation and recalling the Dicke Hamiltonian in natural units $(\hbar=1)$

$$
H_{\mathrm{D}}(\Omega)=\omega a^{\dagger} a+\Delta J^{z}+\frac{\Omega}{\sqrt{N}}\left(a+a^{\dagger}\right) J^{x},
$$

where $a, a^{\dagger}$ are bosonic annihilation and creation operators for a single photonic mode of frequency $\omega$. The $J^{\alpha}$ s $(\alpha=x, y, z)$ are collective variables describing an ensemble of $N$ spin- $\frac{1}{2}$ atoms which effectively behaves like a single larger spin. These operators satisfy the standard $S U(2)$ commutation relations $\left[J^{\alpha}, J^{\beta}\right]=i \epsilon_{\alpha \beta \gamma} J^{\gamma}$ and we work in the largest representation, where $J^{2}=J^{\alpha} J_{\alpha}=$ $N(N+2) / 4$. The parameter $\Delta$ is the energy cost to "flip an atomic spin". The light-matter coupling constant $\Omega$ is divided by $\sqrt{N}$ to ensure that the energy is extensive in the number of atoms.

The Dicke model undergoes a continuous quantum phase transition at $\Omega=\Omega_{c}=\sqrt{\omega \Delta}$. Below $\Omega_{c}$ the system is in the normal phase (NP) and the average density of photons $\left\langle a^{\dagger} a\right\rangle / N$ in the ground state (GS) vanishes in the thermodynamic limit $N \rightarrow \infty$. For $\Omega>\Omega_{c}$, the system is in the superradiant phase (SP) and develops a macroscopic cavity field, i.e. the average density of photons converges to a finite value. Correspondingly, the average expectations $\left\langle a+a^{\dagger}\right\rangle / \sqrt{N}$ and $\left\langle J^{x}\right\rangle / N$ also acquire a finite value, resulting in spontaneous breaking of the $\mathbb{Z}_{2}$ symmetry $U=\mathrm{e}^{i \pi\left(a^{\dagger} a+J^{z}\right)}$. This phase transition has first been studied by Hepp and Lieb ${ }^{20 \mid 3940}$, who computed the full partition function of the model in the thermodynamic limit $N \rightarrow \infty$.

\section{NP AND SP: THE COORDINATE PICTURE}

We work here in the formalism developed in Ref. ${ }^{21}$, which effectively maps the Dicke Hamiltonian onto a twoboson model. This is achieved via the Holstein-Primakoff transformation $J^{z}=b^{\dagger} b-N / 2, \quad J^{+}=b^{\dagger} \sqrt{N-b^{\dagger} b}$, $J^{-}=\left(\sqrt{N-b^{\dagger} b}\right) b$, where $b, b^{\dagger}$ satisfy ordinary bosonic commutation relations. By dropping terms proportional to $1 / N$, in the NP one obtains a quadratic bosonic Hamiltonian $^{21}$ (see also Appendix B)

$$
H_{\mathrm{NP}}(\Omega)=\omega a^{\dagger} a+\Delta b^{\dagger} b+\Omega\left(a+a^{\dagger}\right)\left(b+b^{\dagger}\right)-\frac{N \Delta}{2} .
$$

In the SP both the $a$ and $b$ operators acquire an expectation value $\propto \sqrt{N}$; hence, the expansion of the square roots in the Holstein Primakoff representation must account for this: one defines new operators $c=a+\sqrt{\alpha}$, $d=b-\sqrt{\beta}$ where $\langle c\rangle$ and $\langle d\rangle$ are of order $O(1)$. This

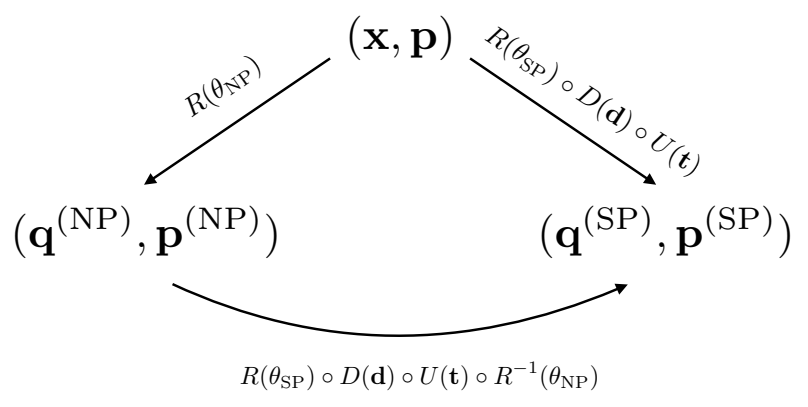

FIG. 1. Diagram of the coordinate transformations that diagonalize the thermodynamic limit of the Dicke Hamiltonian both in the NP and SP. The original pair of canonical coordinates (x, p) in Eqs. 4. 57 are transformed via a rotation $R\left(\theta_{\mathrm{NP}}\right)$ to diagonalize the Hamiltonian in the NP. For the $\mathrm{SP}$ the transformation is more involved, requiring to compose a translation represented by $U(\mathbf{t})$, a dilation $D(\mathbf{d})$ and another rotation $R\left(\theta_{\mathrm{SP}}\right)$. The overall transformation that links NP and SP coordinates is easily obtained by composing successive transformations (see also Appendices B and C). The explicit parameters that enter in the transformations are $\mathbf{t}=\left(\frac{\sqrt{2} \Omega}{\omega} \sqrt{\frac{N\left(1-\mu^{2}\right)}{\omega}},-\sqrt{\frac{N(1-\mu)}{\Delta}}\right), \tan \left(2 \theta_{\mathrm{SP}}\right)=\frac{2 \omega \Delta \mu^{2}}{\mu^{2} \omega^{2}-\Delta^{2}}$, $\tan \left(2 \theta_{\mathrm{NP}}\right)=\frac{4 \Omega \sqrt{\omega \Delta}}{\omega^{2}-\Delta^{2}}$ and $\mathbf{d}=\left(1, \sqrt{\frac{2 \mu}{1+\mu}}\right)$.

yields

$$
\begin{aligned}
H_{\mathrm{SP}} & =\omega c^{\dagger} c+\frac{\Delta(1+\mu)}{2 \mu} d^{\dagger} d+\frac{\Delta(1-\mu)(3+\mu)}{8 \mu(1+\mu)}\left(d^{\dagger}+d\right)^{2} \\
& +\Omega \mu \sqrt{\frac{2}{1+\mu}}\left(c^{\dagger}+c\right)\left(d^{\dagger}+d\right)-\frac{N}{2}\left(\frac{2 \Omega^{2}}{\omega}+\frac{\Delta^{2} \omega}{8 \Omega^{2}}\right)
\end{aligned}
$$

where $\mu=(\omega \Delta) /\left(4 \Omega^{2}\right)=\left(\Omega_{c} / \Omega\right)^{2}, \alpha=(\Omega / \omega) \sqrt{N\left(1-\mu^{2}\right)}$ and $\beta=\sqrt{N(1-\mu) / 2}$.

In the thermodynamic limit, $H_{\mathrm{NP}}$ and $H_{\mathrm{SP}}$ capture, separately in each phase, the thermodynamic properties of the Dicke model. More specifically, one can interpret them as an effective description of the dominating Gaussian fluctuations of the order parameter far from the critical region. As such, this description is rather generic for many-body statistical systems undergoing a discrete symmetry breaking. As shown below, the specific choice of the Dicke model as an example allows us to more easily establish the connection between the relevant fluctuations in the two phases.

Hereafter, we neglect the $1 / N$ corrections in $H_{\mathrm{NP} / \mathrm{SP}}$, which is equivalent to taking the thermodynamic limit $N \rightarrow \infty$ before calculating the time evolution of the system. With this approximation, the quench is mapped onto the non-equilibrium dynamics of a two-dimensional harmonic oscillator (2DHO). We remark that this mapping is not exact, as the discarded corrections could affect the large-deviation properties in the Dicke model, but the $2 \mathrm{DHO}$ is sufficient to highlight non-analyticities in the work rate function. 
Since $H_{\mathrm{NP}}$ and $H_{\mathrm{SP}}$ are quadratic, they can be diagonalized via appropriate generalized Bogolyubov transformations ${ }^{42}$. However, for our purposes it is more convenient to work in the aforementioned 2DHO representation and consider the associated coordinate representation $\mathbf{x}=(x, y), \mathbf{p}=\left(p_{x}, p_{y}\right)$ :

$$
\begin{array}{ll}
x=\frac{1}{\sqrt{2 \omega}}\left(a+a^{\dagger}\right), & p_{x}=i \sqrt{\frac{\omega}{2}}\left(a^{\dagger}-a\right), \\
y=\frac{1}{\sqrt{2 \Delta}}\left(b+b^{\dagger}\right), & p_{y}=i \sqrt{\frac{\Delta}{2}}\left(b^{\dagger}-b\right),
\end{array}
$$

where $\left[x, p_{x}\right]=\left[y, p_{y}\right]=i$ and all other commutators vanish. In each phase, the Bogolyubov transformation which diagonalizes $H_{\mathrm{NP} / \mathrm{SP}}$ becomes a geometric transformation of the coordinates, namely a combination of rotations $R$, dilations $D$ and translations $U$ as shown in Fig. 1. In their diagonal bases, the Hamiltonians are written in terms of new coordinates $\mathbf{q}^{(\nu)}=\left(q_{x}^{(\nu)}, q_{y}^{(\nu)}\right)$, $\mathbf{p}^{(\nu)}=\left(p_{x}^{(\nu)}, p_{y}^{(\nu)}\right)(\nu=\mathrm{NP} / \mathrm{SP})$ and have fundamental frequencies

$$
\omega_{ \pm}^{(\nu)}=\sqrt{\frac{1}{2}\left(\omega^{2}+\frac{\Delta^{2}}{j_{\nu}^{2}} \pm \sqrt{\left(\omega^{2}-\frac{\Delta^{2}}{j_{\nu}^{2}}\right)^{2}+16 j_{\nu} \Omega^{2} \omega \Delta}\right)}
$$

with $j_{\mathrm{NP}}=1$ and $j_{\mathrm{SP}}=\mu$.

\section{LOSCHMIDT AMPLITUDE}

A fundamental quantity which characterizes the work statistics is the Loschmidt amplitude ${ }^{13}$

$$
L(t)=\left\langle\mathrm{GS}\left(\Omega_{0}\right)\left|e^{-i t H_{\mathrm{NP}}(\Omega)}\right| \mathrm{GS}\left(\Omega_{0}\right)\right\rangle,
$$

with $\left|\mathrm{GS}\left(\Omega_{0}\right)\right\rangle$ denoting one of the two superradiant ground states at maximal transverse magnetization, i.e. chosen as the ground state of $H_{\mathrm{SP}}\left(\Omega_{0}\right)+\epsilon J^{x}$ for $\epsilon \rightarrow 0^{+}$ (the other one would be obtained by minimizing the energy for $\epsilon \rightarrow 0^{-}$, corresponding to $\left.J^{x} \rightarrow-J^{x}\right)$. Without loss of generality, we rescale the energies so that the NP ground state has zero energy.

Inserting four completeness relations in (7), the Loschmidt amplitude becomes

$$
\begin{aligned}
L(t)=\int & d^{2} q_{1}^{(\mathrm{SP})} d^{2} q_{2}^{(\mathrm{SP})} d^{2} q_{1}^{(\mathrm{NP})} d^{2} q_{2}^{(\mathrm{NP})}\left\langle\mathrm{GS}\left(\Omega_{0}\right) \mid \mathbf{q}_{1}^{(\mathrm{SP})}\right\rangle \times \\
& \times\left\langle\mathbf{q}_{1}^{(\mathrm{SP})} \mid \mathbf{q}_{1}^{(\mathrm{NP})}\right\rangle\left\langle\mathbf{q}_{1}^{(\mathrm{NP})}\left|e^{-i t H_{\mathrm{NP}}}\right| \mathbf{q}_{2}^{(\mathrm{NP})}\right\rangle \times \\
& \times\left\langle\mathbf{q}_{2}^{(\mathrm{NP})} \mid \mathbf{q}_{2}^{(\mathrm{SP})}\right\rangle\left\langle\mathbf{q}_{2}^{(\mathrm{SP})} \mid \mathrm{GS}\left(\Omega_{0}\right)\right\rangle
\end{aligned}
$$

In the expression above, we note that: (i) $\left\langle\mathrm{GS}\left(\Omega_{0}\right) \mid \mathbf{q}_{1}^{(\mathrm{SP})}\right\rangle$ and $\left\langle\mathbf{q}_{2}^{(\mathrm{SP})} \mid \mathrm{GS}\left(\Omega_{0}\right)\right\rangle$ are the ground state wavefunctions of the SP two-dimensional harmonic oscillator and are therefore (as functions of $\mathbf{q}_{1 / 2}^{(\mathrm{SP})}$ ) Gaussians with zero mean and variances $\left(1 / \sqrt{\omega_{+}^{(\mathrm{SP})}}, 1 / \sqrt{\omega_{-}^{(\mathrm{SP})}}\right)$;

$\left\langle\mathbf{q}_{1}^{(\mathrm{NP})}\left|e^{-i t H_{\mathrm{NP}}}\right| \mathbf{q}_{2}^{(\mathrm{NP})}\right\rangle$ is the propagator of the NP twodimensional oscillator, and thus has a complex Gaussian structure which becomes purely Gaussian after a Wick rotation to imaginary time $t \rightarrow-i s$; (iii) the overlaps $\left\langle\mathbf{q}_{j}^{(\mathrm{NP})} \mid \mathbf{q}_{j}^{(\mathrm{SP})}\right\rangle$ correspond to a change of variable (see Appendices B and $\mathrm{C}$ ) in the integration according to the canonical transformation mapping $\mathbf{q}^{(\mathrm{NP})} \leftrightarrow$ $\mathbf{q}^{(\mathrm{SP})}$ (see Fig. 1), which can be expressed as $\mathbf{q}^{(\mathrm{SP})}=$ $S \mathbf{q}^{(\mathrm{NP})}+\sqrt{N} \mathbf{T}$, where we introduced the shorthand $S=R\left(\theta_{\mathrm{SP}}\right) D(\mathbf{d}) R^{-1}\left(\theta_{\mathrm{NP}}\right)$ and $\sqrt{N} \mathbf{T}=R\left(\theta_{\mathrm{SP}}\right) D(\mathbf{d}) \mathbf{t}$ in relation to the sketch in Fig. 1 The problem of calculating $L(t)$ is now reduced to a Gaussian integration, which can be solved exactly to yield a large deviation form

$$
L(t)=A(t) e^{N l(t)},
$$

where both the function $l(t)$ and the prefactor $A(t)$ are intensive functions, i.e. do not depend on $N$. To write them in a compact form, we introduce three diagonal matrices $Q_{\mathrm{SP}}=\operatorname{diag}\left(\omega_{+}^{(\mathrm{SP})}, \omega_{-}^{(\mathrm{SP})}\right), P_{ \pm}(t)=$ $\pm i \operatorname{diag}\left(\omega_{+}^{(\mathrm{NP})}\left(\tan \left(\frac{\omega_{+}^{(\mathrm{NP})} t}{2}\right)\right)^{ \pm 1}, \omega_{-}^{(\mathrm{NP})}\left(\tan \left(\frac{\omega_{-}^{(\mathrm{NP})} t}{2}\right)\right)^{ \pm 1}\right)$ and

$$
\mathcal{K}_{ \pm}(t)=S^{\top} Q_{\mathrm{SP}} S+P_{ \pm}(t) .
$$

In terms of these matrices, the rate function reads

$$
l(t)=-\mathbf{T}^{\top}\left(Q_{\mathrm{SP}}-Q_{\mathrm{SP}} S \mathcal{K}_{+}^{-1}(t) S^{\top} Q_{\mathrm{SP}}\right) \mathbf{T},
$$

while the prefactor is

$$
A(t)=\sqrt{\frac{-4 \operatorname{det} D^{2} \omega_{+}^{(\mathrm{NP})} \omega_{-}^{(\mathrm{NP})} \omega_{+}^{(\mathrm{SP})} \omega_{-}^{(\mathrm{SP})}}{\sin \left(\omega_{+}^{(\mathrm{NP})} t\right) \sin \left(\omega_{-}^{(\mathrm{NP})} t\right) \operatorname{det} \mathcal{K}_{+}(t) \operatorname{det} \mathcal{K}_{-}(t)}} .
$$

\section{STATISTICS OF WORK}

The Loschmidt amplitude calculated above gives access to the statistics of the work done by the quench $\Omega_{0} \rightarrow \Omega$, as shown in 14 . The average work per atom $w=W_{N} / N$ is a stochastic variable with a distribution $P(w)$ whose generating function is the analytical continuation of $L(t)$ to imaginary time $t \rightarrow-i s$. In the large- $N$ limit, $L(-i s)$ can be written as in Eq. (9) by substitution. The probability $P(w)$ must therefore fulfill a large deviation principle as well, namely $P(w) \propto$ $\exp (-N p(w))$ and furthermore the rate function $p(w)$ and the SCGF $l(-i s)$ are related by a Legendre transform $p(w)=-\inf _{s \in \mathbb{R}}(w s+l(-i s))$ (Gartner-Ellis theorem). These are standard results in large deviation theory and we refer the reader $t \mathrm{C}^{43}$ for a comprehensive review. In the following we investigate the behavior of the rate function $p(w)$ starting from the SCGF $l(-i s)$ in Eq. (11). 


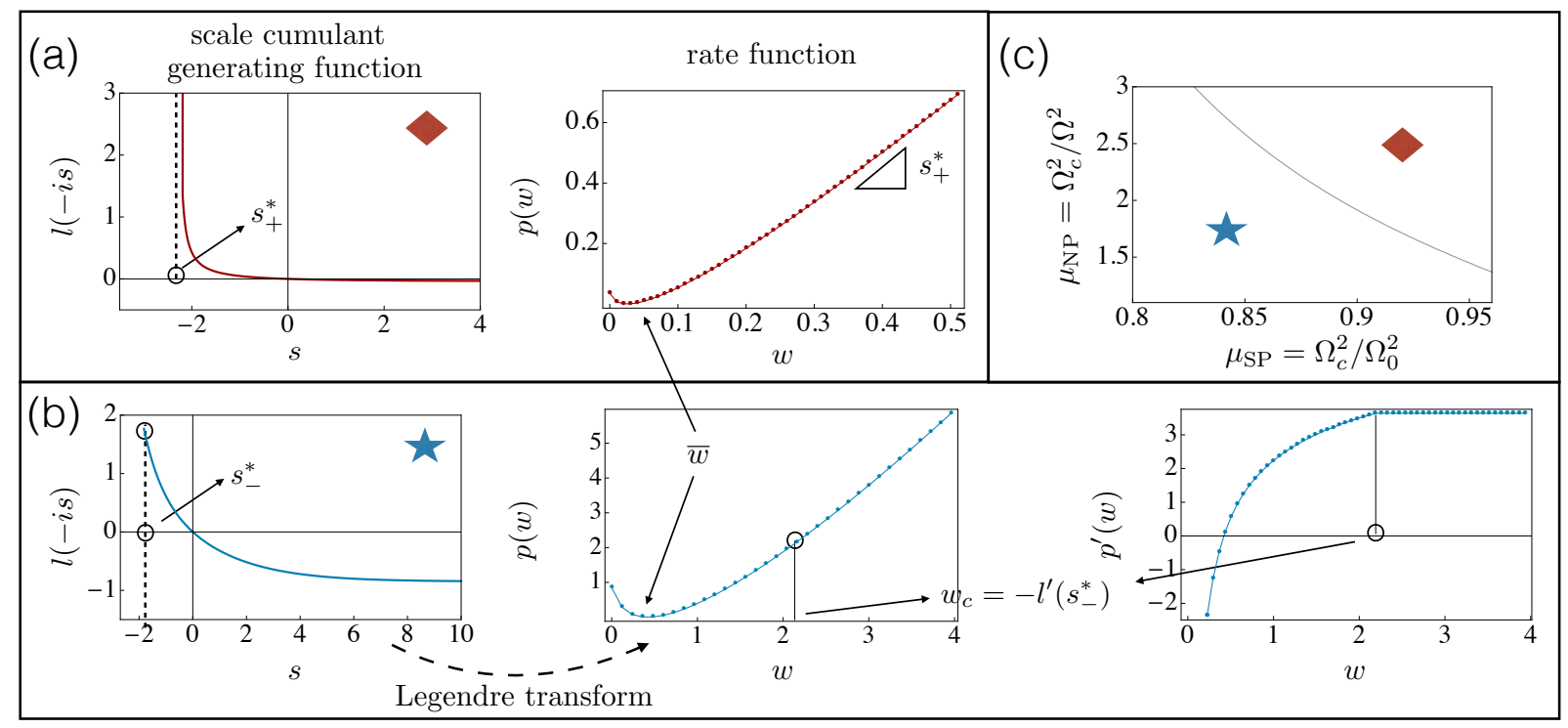

FIG. 2. Phase transitions in the work distribution $p(w)$ obtained as the Legendre transform of the SCGF $l(-i s)$ Eq. 11) (at fixed $\omega=1)$. (a) The SCGF $l(-i s)$ diverges as $s \rightarrow s_{+}^{*}$. Correspondingly, $p(w)$, which is obtained by a Legendre transform (LT) from $l(-i s)$, approaches asymptotically the linear regime with slope $-s_{+}^{*}$ for $w \gg \bar{w}$ (with $\bar{w}$ the typical value of the work, i.e. the one for which $p(\bar{w})=0)$. This scenario is the one expected for the models that belong to the class $\mathrm{B}$ introduced in Ref. 14 (b) The singular behavior of $l(-i s)$ is controlled by $s_{-}^{*}$ which is approached at finite value with finite derivative $l^{\prime}\left(s_{-}^{*}\right)$. The LT thus displays a non-analytical point in $w_{c}=-l^{\prime}\left(-i s_{-}^{*}\right)$ and $p(w)=-s_{-}^{*} w$ for $w>w_{c}$. This non-analytical point of the rate function corresponds to a phase transition in the rare fluctuations of the work. Plots in (a) and (b) are obtained with the same $\Delta=0.8$ and $\Omega=0.3$ by tuning the superradiant coupling from $\Omega_{0}=0.47$ to $\Omega_{0}=0.9$. (c) Analytical (diamond) and non-analytical (star) domains of $p(w)$ in the plane $\mu_{\mathrm{NP}}-\mu_{\mathrm{SP}}$ with $\mu_{\mathrm{NP}}=\Omega_{c}^{2} / \Omega^{2}$ and $\mu_{\mathrm{SP}}=\Omega_{c}^{2} / \Omega_{0}^{2}$ at $\Delta=0.8$. At fixed $\Omega$, the non-analytical point appears only if the quench starts deep enough in the SP. The solid line denotes the boundary between the two areas and is where the non-analyticity in $p(w)$ appears.

The 2DHO model has no upper bound to its energy spectrum and thus should belong to class B, according to Ref. ${ }^{14}$. However, in some parameter regimes its SCGF $l(-i s)$ reaches the leftmost point of its domain with finite (instead of diverging) derivative, see Fig. 2(b). This property of $l(-i s)$ translates in a non-analytical behavior of $p(w)$, which was not previoulsy reported.

The appearance of such a regime is directly related to the domain of $L(-i s)=\left\langle\mathrm{GS}\left(\Omega_{0}\right)\left|e^{-s H_{\mathrm{NP}}(\Omega)}\right| \mathrm{GS}\left(\Omega_{0}\right)\right\rangle$. In performing the analytic continuation of $(9)$, we thereby have to stop at the first singularity encountered. First, we note that for $s \geq 0 L(-i s)$ is always well-defined. Second, a singularity of $L(-i s)$ can be either associated to a singularity of SCGF $l(-i s)$ or of the amplitude $A(-i s)$ (or both). Third, for $l(-i s)$ this can only occur when $\operatorname{det} \mathcal{K}_{+}(-i s)=0$, whereas for $A(-i s)$ singularities can additionally emerge when $\operatorname{det} \mathcal{K}_{-}(-i s)=0$. We denote by $s_{ \pm}^{*}$ the rightmost singular point of $\mathcal{K}_{ \pm}(-i s)$. We remark that $\sin \left(\omega_{+}^{(\mathrm{NP})} t\right) \sin \left(\omega_{-}^{(\mathrm{NP})} t\right) \operatorname{det} \mathcal{K}_{-}(t) \stackrel{t \rightarrow 0}{\rightarrow}$ $\omega_{-}^{(\mathrm{NP})} \omega_{+}^{(\mathrm{NP})} / 4$, curing the singularity in $s=0$ and implying $s_{-}^{*}<0$. The left domain edge of $L(-i s)$ is therefore $\max \left(s_{+}^{*}, s_{-}^{*}\right)$. Two regimes can thus be identified: if $s_{+}^{*}>s_{-}^{*}$, the SCGF $l(-i s)$ diverges at its leftmost point (corresponding to class B of Ref. ${ }^{14}$ ). Correspondingly, $p(w)$ approaches asymptotically a linear regime with slope $-s_{+}^{*}$ for $w \rightarrow+\infty$, as sketched in Fig. 2(a).
If instead $s_{+}^{*}<s_{-}^{*}$, then $l(-i s)$ remains finite and differentiable in $s_{-}^{*}$, signalling a different qualitative behavior. This in fact yields a point of non-analyticity in $p(w)$ located at $w_{c}=-l^{\prime}\left(-i s_{-}^{*}\right)<+\infty$, sketched in Fig. 2(b). For all $w>w_{c}, p(w)=-s_{-}^{*} w-l\left(-i s_{-}^{*}\right)$ exactly, corresponding to a jump in the second derivative of $p(w)$, which vanishes for $w>w_{c}$, see Fig. 2(b). The emergence of either scenario depends on the pre- and post-quench parameters $\Omega_{0}, \Omega, \omega$ and $\Delta$. In Fig. 2(c) we provide an example showing the emergence of these two regions for $\Delta=0.8, \omega=1$ and various values of $\Omega_{0}$ and $\Omega$. These are produced by numerically solving $\operatorname{det} \mathcal{K}_{+}(-i s)=0$.

Finally, we remark that quenches within the NP have already been studied in 29 . In that case, $\log L(t)$ is not proportional to $N$ (extensive) and therefore a large deviation behavior does not emerge. The NP to SP and the SP to SP protocols will be investigated the object of future investigations.

\section{DISCUSSION AND CONCLUSIONS}

We identified singularities in the distribution function of work for a system undergoing a quantum quench, extending the original classification presented in Ref.14. In particular, the rate function describing the statis- 
tics of the work exhibits in this regime a non-analytical point, signaling an out-of-equilibrium phase transition in the rare fluctuations of the work. It is important to stress that the physical intepretation of a non-analytical point in this context is not straightforward and deserves by itself a more detailed and quantitative investigation. Our current intuition is based on similar works in the context of classical stochastic 44 (or more generally, dissipative $47-56$ ) systems. There, non-equilibrium phase transitions in the rare fluctuations of some observable (typically a current) are associated to a sharp change in the nature of the "typical" configurations the system displays when the observable (current) is biased towards values far from its average. Similarly, we think that the qualitative nature of quantum states populated when large fluctuations of the work are encountered may change sharply on the two sides of the transition, but further studies are required to establish whether this is the case.

It would be also interesting to understand what relation there is between the non-analiticities found here and the dynamical phase transitions investigated in $[57$.

Acknowledgements - The research leading to these results has received funding from the European Research Council under the European Unions Seventh Framework Programme (FP/2007-2013)/ERC Grant Agreement No. 335266 (ESCQUMA). P.R. acknowledges funding by the European Union through the H2020 - MCIF No. 766442. I.L. gratefully acknowledges funding through the Royal Society Wolfson Research Merit Award. The authors wish to thank M. Heyl and A. Gambassi for useful discussions at a preliminary stage of the project and $H$. Touchette for useful comments on the manuscript.

\section{A. Dicke model and Holstein-Primakoff transformation}

The exact form in the thermodynamic limit of the ground state of the Dicke model can be worked out both in the normal (NP) and in the superradiant phase (SP) through a suitable Holstein-Primakoff transformation. Let us consider the Dicke Hamiltonian:

$$
H_{\mathrm{D}}=\omega a^{\dagger} a+\Delta J^{z}+\frac{\Omega}{\sqrt{N}}\left(a+a^{\dagger}\right) J^{x}
$$

where the $J^{\alpha}$ 's $(\alpha=x, y, z)$ form an irreducible representation of the angular momentum of dimension $N / 2$. In the large- $N$ limit we can employ the following HolsteinPrimakoff transformation:

$$
J^{z}=b^{\dagger} b-N / 2, \quad J^{+}=b^{\dagger} \sqrt{N-b^{\dagger} b}, \quad J^{-}=\sqrt{N-b^{\dagger} b} b,
$$

where $b, b^{\dagger}$ satisfy ordinary bosonic commutation relations. This leads to:

$$
\begin{aligned}
H_{\mathrm{D}} & =\omega a^{\dagger} a+\Delta b^{\dagger} b-\frac{N \Delta}{2}+ \\
& +\Omega\left(a+a^{\dagger}\right)\left(b^{\dagger} \sqrt{1-\frac{b^{\dagger} b}{N}}+\sqrt{1-\frac{b^{\dagger} b}{N}} b\right) .
\end{aligned}
$$

In the thermodynamic limit we can naively ignore the terms proportional to $1 / \sqrt{N}$. In this way we obtain a solvable quadratic bosonic model. This works in the NP at small $\Omega$, but this approximation breaks down in the SP for $\Omega$ large enough, signaling that a quantum phase transition is taking place. In the following we analyze separately the two different phases obtaining the corresponding effective Hamiltonians. We will focus, in particular, on the coordinate representation, that will be useful to evaluate the Loschmidt amplitudes. The results summarized here in the following two subsections are extensively covered in 21 .

\section{B. Normal phase}

In the $\mathrm{NP}$ we omit the $1 / \sqrt{N}$ terms so that Eq. reduces to

$$
H_{\mathrm{NP}}=\omega a^{\dagger} a+\Delta b^{\dagger} b+\Omega\left(a+a^{\dagger}\right)\left(b+b^{\dagger}\right)-\frac{N \Delta}{2} .
$$

This Hamiltonian can be diagonalized by a suitable Bogoliubov transformation that mixes the four different creation and annihilation operators. However the picture is simpler if we switch to the coordinate space, by writing:

$$
\begin{array}{ll}
x=\frac{1}{\sqrt{2 \omega}}\left(a+a^{\dagger}\right), & p_{x}=i \sqrt{\frac{\omega}{2}}\left(a^{\dagger}-a\right), \\
y=\frac{1}{\sqrt{2 \Delta}}\left(b+b^{\dagger}\right), & p_{y}=i \sqrt{\frac{\Delta}{2}}\left(b^{\dagger}-b\right) .
\end{array}
$$

In this way, it is easy to realize that a rotation in the $(x, y)$-plane puts the Hamiltonian in a diagonal form. In particular we need the following coordinate transformation:

$$
\begin{aligned}
\mathbf{q}^{(\mathrm{NP})} & =\left(q_{x}^{(\mathrm{NP})}, q_{y}^{(\mathrm{NP})}\right)^{\top}=R\left(\theta_{\mathrm{NP}}\right) \mathbf{x} \\
R(\theta) & =\left(\begin{array}{cc}
\cos (\theta) & \sin (\theta) \\
-\sin (\theta) & \cos (\theta)
\end{array}\right)
\end{aligned}
$$

with $\theta_{\mathrm{NP}}$ given by:

$$
\tan \left(2 \theta_{\mathrm{NP}}\right)=\frac{4 \Omega \sqrt{\omega \Delta}}{\omega^{2}-\Delta^{2}}
$$

The eigenfrequencies of the Hamiltonian in the NP read:

$$
\omega_{ \pm}^{(\mathrm{NP})}=\sqrt{\frac{1}{2}\left(\omega^{2}+\Delta^{2} \pm \sqrt{\left(\omega^{2}-\Delta^{2}\right)^{2}+16 \Omega^{2} \omega \Delta}\right)} .
$$


It follows from Eq. 22 that the potential is not bounded from below for $\Omega>\sqrt{\omega \Delta} / 2=\Omega_{c}$, thus signaling that this effective Hamiltonian description breaks down at strong coupling. Before moving to the analysis of the $\mathrm{SP}$, we notice that the ground state of the NP in the coordinate basis $\mathbf{q}^{(\mathrm{NP})}$ is a 2-dimensional Gaussian centered around $\mathbf{q}^{(\mathrm{NP})}=(0,0)$ with variance $\left(\sigma_{x}, \sigma_{y}\right)=$ $\left(1 / \sqrt{\omega_{+}^{(\mathrm{NP})}}, 1 / \sqrt{\omega_{-}^{(\mathrm{NP})}}\right)$.

\section{Superradiant phase}

The derivation of the effective Hamiltonian in the SP is more involved. We refer $t d^{21}$ for the details. Here we only report the fundamental results that we will need in the following to compute the Loschmidt amplitude corresponding to the quench for the NP to the SP. Starting from Eq. (15) we define two new operators $c=a-\sqrt{\alpha}$, $d=b+\sqrt{\beta}$ and we choose the displacement parameters properly in order to eliminate the terms linear in the bosonic operators. In this way we get an effective Hamiltonian for the $\mathrm{SP}$, that reads:

$$
\begin{aligned}
H_{\mathrm{SP}} & =\omega c^{\dagger} c+\frac{\Delta(1+\mu)}{2 \mu} d^{\dagger} d+\frac{\Delta(1-\mu)(3+\mu)}{8 \mu(1+\mu)}\left(d^{\dagger}+d\right)^{2} \\
& +\Omega \mu \sqrt{\frac{2}{1+\mu}}\left(c^{\dagger}+c\right)\left(d^{\dagger}+d\right)+\text { const. }
\end{aligned}
$$

where $\mu=(\omega \Delta) /\left(4 \Omega^{2}\right)=\left(\Omega_{c} / \Omega\right)^{2}$. Again we focus on the coordinate space representation. In order to get the diagonal form of the hamiltonian in the SP, we need to apply three succesive canonical transformations, firstly a translation represented by the vector $\mathbf{t}=\left(t_{x}, t_{y}\right)$ (which is the transformation that allows to get $H_{\mathrm{SP}}$ from Eq. (15)), then a dilation $D(\mathbf{d})$ on the $y$ coordinate and finally a rotation by an angle $\theta_{\mathrm{SP}}$. In formulas:

$$
\mathbf{q}^{(\mathrm{SP})}=R\left(\theta_{\mathrm{SP}}\right) D(\mathbf{d})(\mathbf{x}+\mathbf{t})
$$

where the explicit parameters of the transformation are:

$$
\begin{aligned}
& \mathbf{t}=\left(\frac{\sqrt{2} \Omega}{\omega} \sqrt{\frac{N\left(1-\mu^{2}\right)}{\omega}}, \sqrt{\frac{N(1-\mu)}{\Delta}}\right), \\
& \tan \left(2 \theta_{\mathrm{SP}}\right)=\frac{2 \omega \Delta \mu^{2}}{\mu^{2} \omega^{2}-\Delta^{2}}, \\
& \mathbf{d}=\left(1, \sqrt{\frac{2 \mu}{1+\mu}}\right) .
\end{aligned}
$$

In the new coordinates of Eq. (24) the effective Hamiltonian $H_{\mathrm{SP}}$ is diagonal with eigenfrequencies given by:

$$
\omega_{ \pm}^{(\mathrm{SP})}=\sqrt{\frac{1}{2}\left(\omega^{2}+\frac{\Delta^{2}}{\mu^{2}} \pm \sqrt{\left(\omega^{2}-\frac{\Delta^{2}}{\mu^{2}}\right)^{2}+4 \omega^{2} \Delta^{2}}\right)} .
$$

Again, in the coordinate basis $\mathbf{q}^{(\mathrm{SP})}$ the ground state of the SP is a Gaussian centered around $\mathbf{q}^{(\mathrm{SP})}=(0,0)$ and with variances $\left(\sigma_{x}, \sigma_{y}\right)=\left(1 / \sqrt{\omega_{+}^{(\mathrm{SP})}}, 1 / \sqrt{\omega_{-}^{(\mathrm{SP})}}\right)$.

Finally, combining Eqs. 20 and 24, we obtain the explicit relation between NP and SP coordinates that we extensively use in the main text:

$$
\begin{aligned}
\mathbf{q}^{(\mathrm{SP})} & =S \mathbf{q}^{(\mathrm{NP})}+\sqrt{N} \mathbf{T}, \\
S & =R\left(\theta_{\mathrm{SP}}\right) D(\mathbf{d}) R\left(\theta_{\mathrm{NP}}\right)^{\top}, \\
\sqrt{N} \mathbf{T} & =R\left(\theta_{\mathrm{SP}}\right) D(\mathbf{d}) \mathbf{t} .
\end{aligned}
$$

\section{Harmonic oscillator propagators and Gaussian integrals}

We consider first a standard quantum harmonic oscillator Hamiltonian

$$
H_{H O}=\frac{p^{2}}{2 m}+\frac{m}{2} \omega^{2} q^{2} .
$$

Its propagator, defined on the position basis as

$$
F\left(q_{1}, q_{2}, t\right)=\left\langle q_{1}\left|\mathrm{e}^{-i t H_{H O}}\right| q_{2}\right\rangle,
$$

connects any state $\left|\psi\left(t_{0}\right)\right\rangle$ at time $t_{0}$ with its time-evolved counterpart at time $t_{0}+t$ via the relation

$\psi\left(q_{1}, t_{0}+t\right) \equiv\left\langle q_{1} \mid \psi\left(t_{0}+t\right)\right\rangle=\int_{-\infty}^{+\infty} \mathrm{d} q_{2} F\left(q_{1}, q_{2}, t\right) \psi\left(q_{2}, t_{0}\right)$,

with $\psi(q, t)=\langle q \mid \psi(t)\rangle$ denoting the corresponding wavefunction. The functional form of the propagator is known and reads

$F\left(q_{1}, q_{2}, t\right)=\left(\frac{m \omega}{2 \pi i \hbar \sin (\omega t)}\right)^{\frac{1}{2}} \mathrm{e}^{\frac{i m \omega}{2 \hbar \sin (\omega t)}\left[\left(q_{1}^{2}+q_{2}^{2}\right) \cos (\omega t)-2 q_{1} q_{2}\right]}$.

This formula can be straightforwardly generalized to any set of $n$ uncoupled harmonic oscillators by simply taking the product:

$$
F^{(n)}\left(\mathbf{q}_{1}, \mathbf{q}_{2}, t\right)=\prod_{i} F_{i}\left(q_{1, i}, q_{2, i}, t\right)
$$

In our case, it is sufficient to stop at two, whose coordinates we label $\mathbf{q}=\left(q_{x}, q_{y}\right)^{\top}$, whose masses are set to 1 and whose frequencies are denoted by $\left(\omega_{+}, \omega_{-}\right)$. Working in natural units $(\hbar=1)$ yields

$$
\begin{aligned}
F^{(2)}\left(\mathbf{q}_{1}, \mathbf{q}_{2}, t\right)= & \frac{1}{2 \pi i}\left(\frac{\omega_{+} \omega_{-}}{\sin \left(\omega_{+} t\right) \sin \left(\omega_{-} t\right)}\right)^{\frac{1}{2}} \\
& \times \mathrm{e}^{\frac{i \omega_{+}}{2 \sin \left(\omega_{+} t\right)}\left[\left(q_{1, x}^{2}+q_{2, x}^{2}\right) \cos \left(\omega_{+} t\right)-2 q_{1, x} q_{2, x}\right]} \times \\
& \times \mathrm{e}^{\frac{i \omega_{-}}{2 \sin \left(\omega_{-} t\right)}\left[\left(q_{1, y}^{2}+q_{2, y}^{2}\right) \cos \left(\omega_{-} t\right)-2 q_{1, y} q_{2, y}\right]}
\end{aligned}
$$

which holds for both the NP and SP cases once the parameters are appropriately substituted. Note that the 
form of $F$ is always that of a (complex) Gaussian function, which can also be more compactly expressed as

$$
F^{(2)}\left(\mathbf{q}_{1}, \mathbf{q}_{2}, t\right)=\frac{1}{2 \pi i}\left(\frac{\omega_{+} \omega_{-}}{\sin \left(\omega_{+} t\right) \sin \left(\omega_{-} t\right)}\right)^{\frac{1}{2}} \mathrm{e}^{-\frac{1}{2} \overrightarrow{\mathbf{q}}^{\top} M \overrightarrow{\mathbf{q}}}
$$

with the shorthand $\overrightarrow{\mathbf{q}}^{\top}=\left(\mathbf{q}_{1}^{\top}, \mathbf{q}_{2}^{\top}\right)=\left(q_{1, x}, q_{1, y}, q_{2, x}, q_{2, y}\right)$ and

$$
M=\mathbb{1} \otimes P_{d}+\sigma^{x} \otimes P_{m i x}
$$

where we defined

$$
P_{d}=\left(\begin{array}{cc}
-i \omega_{+} \cot \left(\omega_{+} t\right) & 0 \\
0 & -i \omega_{-} \cot \left(\omega_{-} t\right)
\end{array}\right)
$$

and

$$
P_{\text {mix }}=\left(\begin{array}{cc}
i \omega_{+} \csc \left(\omega_{+} t\right) & 0 \\
0 & i \omega_{-} \csc \left(\omega_{-} t\right)
\end{array}\right)
$$

These expressions can be simplified by performing a rotation on the "1-2" components mapping $\sigma^{x} \rightarrow \sigma^{z}$ in (39), which transforms $M$ into a block diagonal matrix

$$
\left(\begin{array}{c|c}
P_{+} & 0 \\
\hline 0 & P_{-}
\end{array}\right)
$$

with $P_{ \pm}=P_{d} \pm P_{m i x}$ defined as in the main text.

The ground state $|G S\rangle$ of a harmonic oscillator also displays a Gaussian wavefunction and in our case it can be expressed as

$$
\langle\mathbf{q} \mid G S\rangle=\left(\frac{\omega_{+} \omega_{-}}{\pi^{2}}\right)^{\frac{1}{4}} \mathrm{e}^{-\frac{\omega_{+}}{2} q_{x}^{2}-\frac{\omega_{-}}{2} q_{y}^{2}}=\left(\frac{\omega_{+} \omega_{-}}{\pi^{2}}\right)^{\frac{1}{4}} \mathrm{e}^{-\frac{1}{2} \mathbf{q}^{\top} Q \mathbf{q}}
$$

with

$$
Q=\left(\begin{array}{cc}
\omega_{+} & 0 \\
0 & \omega_{-}
\end{array}\right)
$$

The expression of the Loschmidt amplitude is therefore a Gaussian integral which can be reduced to the form

$$
G(A, \mathbf{b})=\int \mathrm{d}^{d} q \mathrm{e}^{-\frac{1}{2} \mathbf{q}^{\top} A \mathbf{q}-\mathbf{b}^{\top} \cdot \mathbf{q}}
$$

with $A$ a $d \times d$ an invertible, diagonalizable matrix whose eigenvalues have positive real part and $\mathbf{b}$ a $d$-dimensional vector. This integral yields

$$
G(A, \mathbf{b})=\frac{1}{(2 \pi)^{\frac{d}{2}} \sqrt{\operatorname{det} A}} \mathrm{e}^{\frac{1}{2} \mathbf{b}^{\top} A^{-1} \mathbf{b}} .
$$

1 L. F. Cugliandolo, eprint arXiv:cond-mat/0210312 (2002), cond-mat/0210312.

2 A. Crisanti and F. Ritort, Journal of Physics A: Mathematical and General 36, R181 (2003)

${ }^{3}$ C. Jarzynski, Annual Review of Condensed Matter Physics 2, 329 (2011), https://doi.org/10.1146/annurevconmatphys-062910-140506

4 J. Berges, S. Borsányi, and C. Wetterich, Phys. Rev. Lett. 93, $142002(2004)$

5 A. Polkovnikov, K. Sengupta, A. Silva, and M. Vengalattore, Rev. Mod. Phys. 83, 863 (2011)

${ }^{6}$ M. Rigol, V. Dunjko, V. Yurovsky, and M. Olshanii, Phys. Rev. Lett. 98, 050405 (2007).

${ }^{\top}$ P. Calabrese, F. H. L. Essler, and M. Fagotti, Phys. Rev. Lett. 106, 227203 (2011)

${ }^{8}$ M. Greiner, O. Mandel, T. W. Hänsch, and I. Bloch, Nature 419, 51 (2002).

9 T. Kinoshita, T. Wenger, and D. S. Weiss, Nature 440, 900 (2006).

10 M. Gring, M. Kuhnert, T. Langen, T. Kitagawa, B. Rauer, M. Schreitl, L. Mazets, D. A. Smith, E. Demler, and J. Schmiedmayer, Science 337, 1318 (2012).

11 M. Esposito, U. Harbola, and S. Mukamel, Rev. Mod. Phys. 81, 1665 (2009).

${ }_{12}$ M. Campisi, P. Hänggi, and P. Talkner, Rev. Mod. Phys. 83, 771 (2011)

is A. Silva, Phys. Rev. Lett. 101, 120603 (2008)

14 A. Gambassi and A. Silva, Phys. Rev. Lett. 109, 250602
(2012)

${ }_{15}$ C. P. Espigares, P. L. Garrido, and P. I. Hurtado, Phys. Rev. E 87, 032115 (2013).

${ }^{10}$ N. Tizón-Escamilla, C. Pérez-Espigares, P. L. Garrido, and P. I. Hurtado, Phys. Rev. Lett. 119, 090602 (2017)

17 R. Dicke, Phys. Rev. 93, 99 (1954).

${ }^{18}$ F. Dimer, B. Estienne, A. S. Parkins, and H. J. Carmichael, Phys. Rev. A 75, 013804 (2007).

19 D. Nagy, G. Kónya, G. Szirmai, and P. Domokos, Phys. Rev. Lett. 104, 130401 (2010).

20 K. Hepp and E. Lieb, Phys. Rev. A 8, 2517 (1973).

21 C. Emary and T. Brandes, Phys. Rev. E 67, 066203 (2003)

${ }^{22}$ K. Baumann, C. Guerlin, F. Brenneke, and T. Esslinger, Nature (London) 464, 1301 (2010).

${ }^{23}$ K. Baumann, R. Mottl, F. Brennecke, and T. Esslinger, Phys. Rev. Lett. 107, 140402 (2011).

24 J. Klinder, H. Keßler, M. Wolke, L. Mathey, and A. Hemmerich, PNAS 112, 3290 (2015).

25 S. Gammelmark and K. Mølmer, New J. Phys. 12, 053035 (2011).

26 L. Bakemeier, A. Alvermann, and H. Fehske, Phys. Rev. A 85, 043821 (2012)

${ }^{2 \tau}$ O. Castaños, E. Nahmad-Achar, R. López-Peña, and J. G. Hirsch, Phys. Rev. A 86, 023814 (2012).

28 M. J. Bhaseen, J. Mayoh, B. D. Simons, and J. Keeling, Phys. Rev. A 85, 013817 (2012).

29 F. N. C. Paraan and A. Silva, Phys. Rev. E 80, 061130 (2009) 
${ }^{30}$ P. Strack and S. Sachdev, Phys. Rev. Lett. 107, 277202 (2011).

${ }^{31}$ M. Buchhold, P. Strack, S. Sachdev, and S. Diehl, Phys. Rev. A 87, 063622 (2013).

${ }_{32}$ P. Rotondo, E. Tesio, and S. Caracciolo, Phys. Rev. B 91, 014415 (2015)

33 S. Gopalakrishnan, B. Lev, and P. Goldbart, Phys. Rev. Lett. 107, 277201 (2011).

34 P. Rotondo, M. Cosentino Lagomarsino, and G. Viola, Phys. Rev. Lett. 114, 143601 (2015)

35 P. Domokos and H. Ritsch, Phys. Rev. Lett. 89, 253003 (2002)

so S. Zippilli, G. Morigi, and H. Ritsch, Phys. Rev. Lett. 93, $123002(2004)$

st J. K. Asbóth, P. Domokos, H. Ritsch, and A. Vukics, Phys. Rev. A 72, 053417 (2005).

38 A. T. Black, H. W. Chan, and V. Vuletić, Phys. Rev. Lett. 91, 203001 (2003)

${ }^{39}$ K. Hepp and E. Lieb, Ann. Phys. 76, 360 (1973).

40 Y. K. Wang and F. T. Hioe, Phys. Rev. A 7, 831 (1973).

41 See Supplementary Material.

42 N. Bogolyubov, J. Phys. (USSR) 11, 23 (1947).

43 H. Touchette, Physics Reports 478, 1 (2009)

44 T. Bodineau and B. Derrida, Phys. Rev. E 72, 066110 (2005)
45 N. Tizón-Escamilla, C. Pérez-Espigares, P. L. Garrido, and P. I. Hurtado, Phys. Rev. Lett. 119, 090602 (2017).

${ }^{46}$ P. I. Hurtado and P. L. Garrido, Phys. Rev. Lett. 107, 180601 (2011)

$4 \overline{4}$ S. Genway, J. M. Hickey, J. P. Garrahan, and A. D. Armour, ArXiv e-prints (2012), arXiv:1212.5200 [condmat.stat-mech]

48 J. M. Hickey, S. Genway, I. Lesanovsky, and J. P. Garrahan, Phys. Rev. B 87, 184303 (2013).

49 J. M. Hickey, S. Genway, and J. P. Garrahan, Phys. Rev. B 89, 054301 (2014).

so J. M. Hickey, ArXiv e-prints (2014), arXiv:1403.5515 [cond-mat.stat-mech],

${ }_{51}$ E. Aghion, D. A. Kessler, and E. Barkai, Phys. Rev. Lett. 118, 260601 (2017).

${ }_{52}$ M. Znidarič, Phys. Rev. Lett. 112, 040602 (2014)

53 D. Manzano and P. I. Hurtado, Phys. Rev. B 90, 125138 (2014)

${ }^{54}$ F. Carollo, J. P. Garrahan, I. Lesanovsky, and C. PérezEspigares, Phys. Rev. E 96, 052118 (2017).

${ }^{55}$ A. Kundu, S. Sabhapandit, and A. Dhar, Journal of Statistical Mechanics: Theory and Experiment 2011, P03007 (2011)

56 S. Sabhapandit, Phys. Rev. E 85, 021108 (2012)

57 M. Heyl, A. Polkovnikov, and S. Kehrein, Phys. Rev. Lett. 110, 135704 (2013). 